\title{
RIGIDITY THEOREMS OF HYPERSURFACES IN LOCALLY SYMMETRIC RIEMANNIAN MANIFOLD
}

\author{
SHICHENG ZHANG AND BAOQIANG WU
}

(Communicated by Lei $\mathrm{Ni}$ )

\begin{abstract}
In this paper, the linear Weingarten hypersurfaces in a locally symmetric Riemannian manifold are investigated and the rigidity theorems are proved by the operator $\square$ introduced by S. Y. Cheng and S. T. Yau, which is a generalization of main results obtained by several authors.
\end{abstract}

\section{INTRODUCTION}

When the ambient manifolds possess very nice symmetry, for example, the sphere, many results have been obtained in the study of the minimal hypersurface and the hypersurface with constant mean curvature or constant scalar curvature in these ambient manifolds. (One can refer to [1-[7]). Recently, Q.M. Cheng and H. Nakagawa [8], and H.W. Xu [9] independently proved the optimal rigidity theorem for the hypersurface of constant mean curvature in a sphere.

In order to study hypersurfaces with constant scalar curvature, Cheng and Yau [12 introduced a new self-adjoint differential operator $\square$ acting on $C^{2}$-functions defined on Riemannian manifolds. As a by-product of this approach they were able to classify closed hypersurface $M$ with constant normalized scalar curvature $R$ satisfying $R \geq c$ and non-negative sectional curvatures immersed in complete and simply connected $(n+1)$-dimensional Riemannian manifolds of constant sectional curvature $c$, which will be denoted by $Q^{n+1}(c)$ and are also known as space forms.

By using the Cheng-Yau technique, X. Liu and H. Li [7] also obtained some rigidity theorems for hypersurface with constant scalar curvature. Therefore, it is important and natural to extend the Riemannian space forms to the locally symmetric Riemannian manifolds.

Let the ambient manifold $N^{n+1}$ be a locally symmetric Riemannian manifold with sectional curvature $K_{N}$ and $M$ be an $n$-dimensional complete hypersurface with constant mean curvature $H$ in $N^{n+1}$. When $\frac{1}{2}<\delta \leq K_{N} \leq 1$ ( $\delta$ is a constant) at all points $x \in M$ and the squared norm of the second fundamental form $S$ satisfies $S<n+\frac{n^{3}}{2(n-1) H^{2}}-\frac{n(n-2)}{2(n-1)} \sqrt{n^{2} H^{4}+4(n-1) H^{2}}$, S. Shu 13] and S. Ding [14] have obtained that the hypersurface $M$ is a totally umbilical hypersurface,

Received by the editors January 10, 2012.

2010 Mathematics Subject Classification. Primary 53B20, 53C24, 53C20.

Key words and phrases. Locally symmetric, linear Weingarten hypersurfaces, totally umbilical.

This work was supported by the National Natural Science Foundation of China (No. 10871218, No. 61271002 and No. 10932002) and the Natural Science Foundation of Xuzhou Normal University (No. 11XLR36). 
respectively. H.W. Xu [15] has also obtained the same result when $M$ is an $n$ dimensional closed minimal hypersurface with constant mean curvature $H$ in $N^{n+1}$ and sectional curvature $K_{N}$ satisfying the condition $\delta \leq K_{N} \leq 1$ at all points $x \in M$ and the squared norm of the second fundamental form $S$ satisfies $S \leq(2 \delta-1) n$.

Now let us introduce the notion of linear Weingarten hypersurfaces in an $(n+1)$ dimensional locally symmetric Riemannian manifold $N^{n+1}$ as follows:

Definition 1.1. Let $M$ be a hypersurface in an $(n+1)$-dimensional locally symmetric Riemannian manifold $N^{n+1}$. We call $M$ a linear Weingarten hypersurface if $c R+d H+e=0$, where $c, d$ and $e$ are constants such that $c^{2}+d^{2} \neq 0, R$ and $H$ respectively denote the scalar curvature and the mean curvature of $M$.

Remark 1.1. When the constant $d=0$ in Definition 1.1, a linear Weingarten hypersurface $M$ reduces to a hypersurface with constant scalar curvature. When the constant $c=0$ in Definition 1.1, a linear Weingarten hypersurface $M$ reduces to a hypersurface with constant mean curvature. In such a sense, the linear Weingarten hypersurfaces can be regarded as a natural generalization of hypersurfaces with constant scalar curvature or with constant mean curvature.

In this paper, let the ambient manifold $N^{n+1}$ be a locally symmetric Riemannian manifold with sectional curvature $K_{N}$ satisfying the condition $K(u \wedge v) \geq c_{2}\left(c_{2}\right.$ is a constant), where $u, v \in T M$ and $K(\omega \wedge v)=c_{1}$ ( $c_{1}$ is a constant), where $\omega \in T^{\perp} M$ and $v \in T M$; we shall say the manifold $N^{n+1}$ satisfies condition (*). We study the linear Weingarten hypersurfaces in a locally symmetric Riemannian manifold.

As in the computation of $([10,11])$, we compute the scalar curvature of a point in $N^{n+1}$,

$$
K=\sum_{A=1}^{n+1} K_{A A}=2 \sum_{i=1}^{n} K_{n+1 i n+1 i}+\sum_{i, j=1}^{n} K_{i j j i}=2 n c_{1}+\sum_{i, j=1}^{n} K_{i j j i} .
$$

It is known that $K$ is constant when $N^{n+1}$ is locally symmetric, so $\sum_{i, j=1}^{n} K_{i j j i}$ is constant.

From (2.3), we denote

$$
n(n-1) P=n^{2} H^{2}-S=n(n-1) r-\sum_{i, j=1}^{n} K_{i j j i},
$$

where $r$ is a normalized scalar curvature of $M$. By investigating Cheng and Yau's operator $\square$ given in $[12$ and using some new estimations, we are able to study the compact or complete linear Weingarten hypersurfaces in a locally symmetric Riemannian manifold as follows:

Theorem 1.1. Let $M$ be an n-dimensional compact hypersurface in a locally symmetric Riemannian manifold $N^{n+1}$ satisfying condition $(*)$. If the squared norm of the second fundamental form $S$ and the mean curvature $H$ satisfy the conditions

$$
n\left(\frac{P-b}{a}\right)^{2} \leq S \leq \frac{n}{(n-2)(n P+2 c)}\left[n(n-1) P^{2}+4 c(n-1) P+n c^{2}\right]
$$


and $P=a H+b,(n-1) a^{2}+4 n b \geq 0$, then

(1) $S=n\left(\frac{P-b}{a}\right)^{2}$ and $M$ is a totally umbilical hypersurface or

(2) $M$ is an isoparametric hypersurface with two distinct principal curvatures, one of which is simple, and $S=\frac{n}{(n-2)(n P+2 c)}\left[n(n-1) P^{2}+4 c(n-1) P+n c^{2}\right]$, where $c=2 c_{2}-c_{1} \geq 0$.

Remark 1.2. Since $n(n-1) P=n(n-1) r-\sum_{i, j=1}^{n} K_{i j j i}$, a hypersurface $M$ in Theorem 1.1 satisfying $P=a H+b$ is just a linear Weingarten hypersurface in Definition 1.1.

Remark 1.3. If $c_{1}=1, c_{2}=1$, i.e. the locally symmetric Riemannian manifold $N^{n+1}$ is the unit sphere $S^{n+1}(1)$, our Theorem 1.1 reduces to Theorem 1.3 in [16].

Remark 1.4. If $c_{1}=1, c_{2}=1$, when the constant $a$ in the above identically vanishes, our Theorem 1.1 reduces to Theorem 2 in [17] and the main theorem in [7.

Theorem 1.2. Let $M$ be an n-dimensional complete hypersurface in a locally symmetric Riemannian manifold $N^{n+1}$ satisfying condition $(*)$. If the squared norm of the second fundamental form $S$ satisfies the conditions $S<2 \sqrt{n-1}$ c and $P=a H+b, a \leq 0,(n-1) a^{2}+4 n b \geq 0$, then $S=n H^{2}$ and $M$ is a totally umbilical hypersurface, where $c=2 c_{2}-c_{1} \geq 0$.

Example. We consider the Riemannian product manifold

$$
N^{n+1}=S^{n}(r) \times R^{1}=\left\{\left(x^{1}, x^{2}, \cdots, x^{n+2}\right) \in R^{n+2}, \sum_{A=1}^{n+1}\left(x^{A}\right)^{2}=r^{2}\right\} .
$$

Its sectional curvature is given by

$$
K\left(u_{i}, u_{j}\right)=\frac{1}{r^{2}}, K\left(u_{i}, u_{n+1}\right)=0 \quad(i, j=1, \cdots, n) .
$$

Then,

$$
M_{1}=\left\{\left(x^{1}, x^{2}, \cdots, x^{n+1}, c\right) \in R^{n+2}\right\} \subset N^{n+1}
$$

is a totally umbilical hypersurface in a locally symmetric Riemannian manifold $N^{n+1}$, and

$$
M_{2}=\left\{\left(0, x^{2}, \cdots, x^{n+2}\right) \in R^{n+2}\right\} \subset N^{n+1}
$$

is an isoparametric hypersurface in a locally symmetric Riemannian manifold $N^{n+1}$, respectively.

\section{Preliminaries}

If $M$ is a hypersurface in $N^{n+1}$, let $\left\{e_{1}, e_{2}, \cdots, e_{n+1}\right\}$ be a local frame of orthonormal vector fields in $N^{n+1}$ such that, restricted to $M$, the vectors $\left\{e_{1}, e_{2}, \cdots\right.$, $\left.e_{n}\right\}$ are tangent to $M$ and the vector $e_{n+1}$ is normal to $M$. Let $\left\{\omega^{1}, \omega^{2}, \cdots, \omega^{n+1}\right\}$ be its dual frame field. We use the following convention on the range of indices:

$$
1 \leq A, B, C, \cdots \leq n+1, \quad 1 \leq i, j, k, \cdots \leq n .
$$

Let $K_{A B C D}$ and $R_{i j k l}$ be the components of the curvature tensors of $N^{n+1}$ and $M$, respectively. Let $h=h_{i j}$ be the second fundamental form of $M$; the square of the norm of $h$ is denoted by $S=\sum_{i, j=1}^{n}\left(h_{i j}\right)^{2}$. 
It is well known that for an arbitrary hypersurface $M$ of $N^{n+1}$, we have

$$
\begin{gathered}
d \omega_{i j}=-\sum_{k} \omega_{i k} \wedge \omega_{k j}+\frac{1}{2} \sum_{k, l} R_{i j k l} \omega^{k} \wedge \omega^{l}, \\
R_{i j k l}=K_{i j k l}+h_{i k} h_{j l}-h_{i l} h_{j k} .
\end{gathered}
$$

The components $R_{i j}$ of Ricci tensor and the normalized scalar curvature $r$ of $M$ are given by

$$
\begin{array}{r}
R_{i j}=\sum_{k=1}^{n} K_{k i j k}+n H h_{i j}-\sum_{k=1}^{n} h_{i k} h_{k j}, \\
n(n-1) r=\sum_{i, j=1}^{n} K_{i j j i}+(n H)^{2}-S .
\end{array}
$$

We denote the first and second covariant derivatives of $h_{i j}$ by $h_{i j k}$ and $h_{i j k l}$ respectively, which are defined as in [1]. Following [1] and 19, we have

$$
\begin{gathered}
h_{i j k}-h_{i k j}=-K_{n+1 i j k}, \\
\sum_{k} h_{i j k} \omega_{k}=d h_{i j}-\sum_{k} h_{i k} \omega_{k j}-\sum_{k} h_{k j} \omega_{k i},
\end{gathered}
$$

and the Ricci formula

$$
h_{i j k l}-h_{i j l k}=\sum_{s} h_{s j} R_{s i k l}+\sum_{s} h_{i s} R_{s j k l} .
$$

Let $K_{n+1 i j k, l}$ be the covariant derivative of $K_{n+1 i j k}$ as the section of $T^{\perp} M \otimes$ $T^{*} M \otimes T^{*} M \otimes T^{*} M$ and let $K_{A B C D, E}$ be the covariant derivative of $K_{A B C D}$ as the curvature tensor of $N^{n+1}$. Restricted to $M$ we have

$$
\sum_{l} K_{n+1 i j k l} \omega_{l}=d K_{n+1 i j k}+\sum_{s} K_{n+1 s j k} \omega_{i s}+\sum_{s} K_{n+1 i j s} \omega_{k s}
$$

and

$$
K_{n+1 i j k, l}=K_{n+1 i j k l}-K_{n+1 i n+1 k} h_{j l}-K_{n+1 i j n+1} h_{k l}+\sum_{m} K_{m i j k} h_{m l} .
$$

The mean curvature of $M$ is given by $H=\frac{1}{n} \sum_{i=1}^{n} h_{i i} e_{n+1}$, and the Laplacian $\Delta h_{i j}$ of the second fundamental form $h$ of $M$ is defined by $\Delta h_{i j}=\sum_{k=1}^{n} h_{i j k k}$ :

$$
\begin{aligned}
\Delta h_{i j}= & \sum_{k} h_{k k i j}+n H K_{n+1 i n+1 j}-\sum_{k} K_{n+1 k n+1 k} h_{i j}+n H \sum_{k} h_{i k} h_{k j}-S h_{i j} \\
& +\sum_{k}\left(K_{l k i k} h_{l j}+K_{l k j k} h_{l i}+2 K_{l i j k} h_{l k}\right)-\sum_{k}\left(K_{n+1 i j k, k}+K_{n+1 k i k, j}\right) .
\end{aligned}
$$

Since $N^{n+1}$ is complete and locally symmetric, we have

$$
K_{A B C D, E}=0,
$$


for all $A, B, C, D, E$. This together with $(2.2),(2.7)$ and (2.8) implies

$$
\begin{aligned}
\frac{1}{2} \Delta S= & \sum_{i, j, k} h_{i j k}^{2}+\sum_{i, j, k} h_{i j k k} h_{i j} \\
= & n H \operatorname{tr} H_{n+1}^{3}-S^{2}+\sum_{i, j, k} h_{i j k}^{2}+\sum_{i, j, k, l} 2\left(h_{i j} h_{k l} K_{l i j k}+h_{l i} h_{i j} K_{l k j k}\right) \\
& +\sum_{i, j, k} h_{i j k k} h_{i j}+\sum_{i, j} n H h_{i j} K_{n+1 i j n+1}-S \sum_{k} K_{n+1 k n+1 k} .
\end{aligned}
$$

Following Cheng-Yau [12, we introduce a modified operator $\square$ acting on any $C^{2}$-function $f$ by

$$
\square f=\sum_{i, j}\left(n H \delta_{i j}-h_{i j}\right) f_{i j}-\frac{n-1}{2} a \Delta f .
$$

It follows [12] that the operator $\square$ is self-adjoint relative to the $L^{2}$ inner product of $M$, i.e.

$$
\int_{M} f \square g=\int_{M} g \square f .
$$

Choose a local frame of orthonormal vector fields $\left\{e_{i}\right\}$ so that at an arbitrary point $x$ of $M, h_{i j}=\lambda_{i} \delta_{i j}$; then at the point $x$, and by use of (1.1) and (2.10), we have

$$
\begin{aligned}
\square(n H) & =n H \Delta(n H)-\sum_{l} \lambda_{i}(n H)_{i i}-\frac{n-1}{2} a \Delta(n H) \\
& =\frac{1}{2} \Delta\left(n(n-1) r-\sum_{i, j=1}^{n} K_{i j j i}\right)+\frac{1}{2} \Delta S \\
& -n^{2}|\nabla H|^{2}-\sum_{i} \lambda_{i}(n H)_{i i}-\frac{1}{2} \Delta(n(n-1) r) \\
& =\frac{1}{2} \Delta S-n^{2}|\nabla H|^{2}-\sum_{i} \lambda_{i}(n H)_{i i} .
\end{aligned}
$$

Putting (2.10) into (2.12), we obtain

$$
\begin{aligned}
\square(n H) & =n H \Delta(n H)-\sum_{l} \lambda_{i}(n H)_{i i} \\
& =\sum_{i, j, k} h_{i j k}^{2}-n^{2}|\nabla H|^{2}+X+Y+Z,
\end{aligned}
$$

where

$$
\begin{aligned}
X & =n H \operatorname{tr} H_{n+1}^{3}-S^{2}, \\
Y & =\sum_{i, j, k, l} 2\left(h_{i j} h_{k l} K_{l i j k}+h_{l i} h_{i j} K_{l k j k}\right), \\
Z & =\sum_{i, j} n H h_{i j} K_{n+1 i j n+1}-S \sum_{k} K_{n+1 k n+1 k} .
\end{aligned}
$$




\section{Proof of theorems}

The following lemmas are useful in the proofs of Theorems 1.1 and 1.2.

Lemma 3.1. Let $M$ be an n-dimensional compact hypersurface in a locally symmetric Riemannian Manifold $N^{n+1}$ satisfying condition (*). If $P=a H+b, a, b \in R$ and $(n-1) a^{2}+4 n b \geq 0$, then we have

$$
|\nabla h|^{2}=\sum_{\alpha, i, j, k}\left(h_{i j k}^{\alpha}\right)^{2} \geq n^{2}|\nabla H|^{2} .
$$

Proof. From the Gauss equation, we have

$$
S=n^{2} H^{2}-n(n-1) P=n^{2} H^{2}-n(n-1)(a H+b) .
$$

Taking the covariant derivative of the above equation, we have

$$
2 \sum_{\alpha, i, j} h_{i j}^{\alpha} h_{i j k}^{\alpha}=2 n^{2} H H_{k}-n(n-1) a H_{k} .
$$

Therefore,

$$
4|h|^{2}|\nabla h|^{2} \geq 4 \sum_{k}\left(h_{i j}^{\alpha} h_{i j k}^{\alpha}\right)^{2}=\left[2 n^{2} H-n(n-1) a\right]^{2}|\nabla H|^{2} .
$$

On the other hand,

$$
\begin{aligned}
{\left[2 n^{2} H-\right.} & n(n-1) a]^{2}-4 n^{2} S \\
= & 4 n^{4} H^{2}+n^{2}(n-1)^{2} a^{2}-4 n^{3}(n-1) H a \\
& -4 n^{3}\left[n H^{2}-(n-1)(a H+b)\right] \\
= & n^{2}(n-1)\left[(n-1) a^{2}+4 n b\right] \\
\geq & 0 .
\end{aligned}
$$

It follows that

$$
|\nabla h|^{2} \geq n^{2}|\nabla H|^{2}
$$

Lemma 3.2 ([19]). Let $\mu_{1}, \cdots, \mu_{n}$ be real numbers such that $\sum_{i} \mu_{i}=0$ and $\sum_{i} \mu_{i}^{2}=B$, where $B=$ const. $\geq 0$. Then

$$
\left|\sum_{i} \mu_{i}^{3}\right| \leq \frac{n-2}{\sqrt{n(n-1)}} B^{\frac{3}{2}}
$$

and equality holds if and only if

$$
\mu_{1}=\cdots=\mu_{n-1}=-\sqrt{\frac{1}{n(n-1)} B}, \mu_{n}=\sqrt{\frac{n-1}{n} B} .
$$

Choose a local frame of orthonormal vector fields $\left\{e_{i}\right\}$ so that at an arbitrary point $x$ of $M, h_{i j}=\lambda_{i} \delta_{i j}$; then at the point $x$ we have

$$
S=\sum_{i} \lambda_{i}^{2}
$$

Putting $\mu_{j}=H-\lambda_{j}$, we obtain

$$
\sum_{j} \mu_{j}=0, \quad|\phi|^{2}=\sum_{j} \mu_{j}^{2}=S-n H^{2},
$$


and

$$
\sum_{i} \lambda_{i}^{3}=n H^{3}+3 H \sum_{i} \mu_{i}^{2}-\sum_{i} \mu_{i}^{3}
$$

So

$$
\begin{aligned}
n H \operatorname{tr} H_{n+1}^{3} & =n H\left(n H^{3}+3 H \sum_{i} \mu_{i}^{2}-\sum_{i} \mu_{i}^{3}\right) \\
& \geq 3 n H^{2}|\phi|^{2}+n^{2} H^{4}-n|H| \frac{n-2}{\sqrt{n(n-1)}}|\phi|^{3},
\end{aligned}
$$

and we obtain

Lemma 3.3. $X \geq|\phi|^{2}\left[n H^{2}-|\phi|^{2}-\frac{n(n-2)}{\sqrt{n(n-1)}}|H||\phi|\right]$.

Lemma 3.4 ([15]). $Y \geq 2 n c_{2}|\phi|^{2}$.

Lemma 3.5. $Z=c_{1} n\left(n H^{2}-S\right)=-c_{1} n|\phi|^{2}$.

Proof.

$$
\begin{aligned}
Z & =\sum_{i, j} n H h_{i j} K_{n+1 i j n+1}-S \sum_{k} K_{n+1 k n+1 k} \\
& =n H \sum_{i} \lambda_{i} K_{n+1 i n+1 i}-S \sum_{i} K_{n+1 i n+1 i} \\
& =\sum_{i}\left(S-n H \lambda_{i}\right) K_{n+1 i n+1 i}=c_{1} n\left(n H^{2}-S\right) .
\end{aligned}
$$

This proves Lemma 3.5.

From the assumption of Theorem 1.1 and Lemmas 3.1, 3.3, 3.4 and 3.5, we obtain

$$
\square(n H) \geq|\phi|^{2}\left[n c-|\phi|^{2}-n|H| \frac{n-2}{\sqrt{n(n-1)}}|\phi|+n H^{2}\right],
$$

where $c=2 c_{2}-c_{1}$.

The next lemmas have essential roles in the proof of Theorem 1.2.

Lemma 3.6 ([20]). Let $M$ be an $n$-dimensional complete Riemannian manifold whose sectional curvature is bounded from below and let $f: M \longrightarrow R$ be a smooth function which is bounded from above on $M$. Then there is a sequence of points $\left\{p_{k}\right\}$ in $M$ such that

$$
\lim _{k \rightarrow \infty} f\left(p_{k}\right)=\sup f ; \lim _{k \rightarrow \infty}\left|\nabla f\left(p_{k}\right)\right|=0 ; \lim _{k \rightarrow \infty} \sup \left(\Delta f\left(p_{k}\right)\right) \leq 0 .
$$

Lemma 3.7. Let $M$ be a complete hypersurface in an $(n+1)$-dimensional locally symmetric Riemannian manifold $N^{n+1}$ with bounded mean curvature. If $P=a H+$ $b,(n-1) a^{2}+4 n b \geq 0$, then there is a sequence of points $\left\{p_{k}\right\} \in M$ such that

$$
\lim _{k \rightarrow \infty} n H\left(p_{k}\right)=n \sup H ; \lim _{k \rightarrow \infty}\left|\nabla n H\left(p_{k}\right)\right|=0 ; \lim _{k \rightarrow \infty} \sup \left(\square(n H)\left(p_{k}\right)\right) \leq 0 .
$$

Proof. Choose a local orthonormal frame field $\left\{e_{1}, e_{2}, \cdots, e_{n}\right\}$ at $p \in M$ such that $h_{i j}=\lambda_{j} \delta_{i j}$. Then,

$$
\square(n H)=\sum_{i}\left(\left(n H-\lambda_{i}^{n+1}\right)-\frac{n-1}{2} a\right)(n H)_{i i} .
$$


If $H \equiv 0$, Theorem 1.1 is obvious. Let us suppose that $H$ is not identically zero. By changing the orientation of $M$ if necessary, we may assume $H>0$.

From (1.1), we have

$$
\begin{aligned}
& n(n-1) P=n^{2} H^{2}-S \\
\left(\lambda_{i}^{n+1}\right)^{2} \leq & S=n^{2} H^{2}-n(n-1)(a H+b) \\
= & (n H)^{2}-(n-1) a(n H)-n(n-1) b \\
= & \left(n H-\frac{1}{2}(n-1) a\right)^{2}-\frac{1}{4}(n-1)\left((n-1) a^{2}+4 n b\right) \\
\leq & \left(n H-\frac{1}{2}(n-1) a\right)^{2}
\end{aligned}
$$

and so

$$
\left|\lambda_{i}^{n+1}\right| \leq\left|n H-\frac{1}{2}(n-1) a\right| .
$$

From (2.2) and (3.4), we show that

$$
R_{i j i j}=K_{i j i j}+h_{i i}^{n+1} h_{j j}^{n+1}-\left(h_{i j}^{n+1}\right)^{2} \geq c_{2}-\left(n H-\frac{1}{2}(n-1) a\right)^{2} .
$$

Since $H$ is bounded, we may apply Lemma 3.6 to $n H$, obtaining a sequence of points $\left\{p_{k}\right\} \in M$ such that

$$
\lim _{k \rightarrow \infty} n H\left(p_{k}\right)=n \sup H, \lim _{k \rightarrow \infty}\left|\nabla n H\left(p_{k}\right)\right|=0 \text { and } \lim _{k \rightarrow \infty} \sup \left((n H)_{i i}\left(p_{k}\right)\right) \leq 0 .
$$

Since $H$ is bounded, taking subsequences if necessary, we can arrive at a sequence $\left\{p_{k}\right\} \in M$ and such that $H\left(p_{k}\right) \geq 0$. Thus from (3.4), we get

$$
\begin{aligned}
0 & \leq n H\left(p_{k}\right)-\frac{1}{2}(n-1) a-\left|\lambda_{i}^{n+1}\right| \\
& \leq n H\left(p_{k}\right)-\frac{1}{2}(n-1) a-\lambda_{i}^{n+1} \\
& \leq n H\left(p_{k}\right)-\frac{1}{2}(n-1) a+\left|\lambda_{i}^{n+1}\right| \\
& \leq 2 n H\left(p_{k}\right)-(n-1) a .
\end{aligned}
$$

Using once more the fact that $H$ is bounded, from (3.6) and $a \leq 0$ we infer that $n H\left(p_{k}\right)-\frac{1}{2}(n-1) a-\lambda_{i}^{n+1}$ is non-negative and bounded. By applying $\square(n H)$ at $p_{k}$, taking the limit and using (3.5) and (3.6), we have

$$
\begin{aligned}
\lim _{k \rightarrow \infty} \sup \left(\square(n H)\left(p_{k}\right)\right) & \left.\leq \sum_{i} \lim _{k \rightarrow \infty} \sup \left[(n H)\left(p_{k}\right)-\frac{1}{2}(n-1) a\right)-\lambda_{i}^{n+1}\right](n H)_{i i}\left(p_{k}\right) \\
& \leq 0 .
\end{aligned}
$$

Proof of Theorem 1.1. Since $M$ is a compact hypersurface in locally symmetric Riemannian manifold $N^{n+1}$, from (3.3) we have

$$
0 \geq \int_{M}\left(S-n H^{2}\right)\left[n c-\left(S-n H^{2}\right)-n|H| \frac{n-2}{\sqrt{n(n-1)}} \sqrt{S-n H^{2}}+n H^{2}\right] d v
$$


On the other hand, since

$$
S \leq \frac{n}{(n-2)(n P+2 c)}\left[n(n-1) P^{2}+4 c(n-1) P+n c^{2}\right]
$$

we get

$$
\frac{n-2}{n} \sqrt{(n(n-1) P+S)(S-n P)} \leq n c+2(n-1) P+\frac{n-2}{n} S .
$$

From (1.1) and (3.1), we have

$$
S-n H^{2}=\frac{n-1}{n}(S-n P) .
$$

So, we obtain

$$
\begin{aligned}
& n c-n H^{2}+\left(S-n H^{2}\right)-\frac{n(n-2)}{\sqrt{n(n-1)}}|H| \sqrt{S-n H^{2}} \\
& =n c+2(n-1) P+\frac{n-2}{n} S-\frac{n-2}{n} \sqrt{(n(n-1) P+S)(S-n P)} .
\end{aligned}
$$

This implies that

$$
\left(S-n H^{2}\right)\left[n c-\left(S-n H^{2}\right)-n|H| \frac{n-2}{\sqrt{n(n-1)}} \sqrt{S-n H^{2}}+n H^{2}\right] \geq 0 .
$$

By (3.4) and (3.7), we obtain either $S-n H^{2}=S-n\left(\frac{P-b}{a}\right)^{2}=0$ and $M$ is totally umbilical or $S=\frac{n}{(n-2)(n P+2 c)}\left[n(n-1) P^{2}+4 c(n-1) P+n c^{2}\right]$.

If $M$ is not totally umbilical, we can see that $S=\frac{n}{(n-2)(n P+2 c)}\left[n(n-1) P^{2}+\right.$ $4 c(n-1) P+n c^{2}$. Hence equalities hold in Lemma 3.1 and Lemma 3.2, and it follows that $\lambda_{i}=$ const. for all $i$ and $(n-1)$ of the $\lambda_{i}$ 's are equal. After re-numbering if necessary, we can assume that

$$
\lambda_{1}=\lambda_{2}=\cdots=\lambda_{n-1}, \lambda_{1} \neq \lambda_{n} .
$$

Therefore, $M$ is an isoparametric hypersurface with two distinct principal curvatures, one of which is simple. This completes the proof of Theorem 1.1.

Proof of Theorem 1.2. Now we assume $P=a H+b$ and $(n-1) a^{2}+4 n b \geq 0$. For a real number $d=\frac{n+2 \sqrt{n-1}}{n-2} \sqrt{n}>0$, we have

$$
2|H||\phi| \leq d H^{2}+\frac{1}{d}|\phi|^{2} .
$$

From (3.3) and (3.11), we obtain

$$
\begin{array}{r}
\square(n H) \geq|\phi|^{2}\left[n c+n H^{2}\left(2-\frac{(n-2) d}{2 \sqrt{n(n-1)}}+\frac{n(n-2)}{2 \sqrt{n(n-1)} d}\right)\right. \\
\left.-S\left(1+\frac{n(n-2)}{2 \sqrt{n(n-1)} d}\right)\right],
\end{array}
$$

that is,

$$
\square(n H) \geq|\phi|^{2}\left(n c-\frac{n}{2 \sqrt{n-1}} S\right) .
$$

From the assumption $S<2 \sqrt{n-1} c$ and according to Lemma 3.7, there exists a sequence of points $\left\{p_{k}\right\}$ in $M$ such that

$$
\lim _{k \rightarrow \infty} \sup \left(\square(n H)\left(p_{k}\right)\right) \leq 0, \lim _{k \rightarrow \infty} n H\left(p_{k}\right)=\sup (n H) .
$$


From (1.1) and (3.1), we have

$$
|\phi|^{2}=n(n-1)\left(H^{2}-a H-b\right) .
$$

Notice that $\lim _{k \rightarrow \infty} n H\left(p_{k}\right)=\sup (n H)$, so we have

$$
\lim _{k \rightarrow \infty}|\phi|^{2}\left(p_{k}\right)=\sup |\phi|^{2}, \lim _{k \rightarrow \infty} S\left(p_{k}\right)=\sup S .
$$

Evaluating (3.12) at the points $p_{k}$ of the sequence, taking the limit and using (3.13), we obtain

$$
0 \geq \lim _{k \rightarrow \infty} \sup \left(\square(n H)\left(p_{k}\right)\right) \geq \sup |\phi|^{2}\left(n c-\frac{n}{2 \sqrt{n-1}} \sup S\right) .
$$

If $\sup S<2 \sqrt{n-1} c$, then we have $\sup |\phi|^{2}=0$, that is, $|\phi|^{2}=0$. Thus, we infer that $S=n H^{2}$ and $M$ is a totally umbilical hypersurface.

This completes the proof of Theorem 1.2.

\section{REFERENCES}

[1] Q. M. Cheng, Susumu Ishikawa, Characterization of the Clifford torus, Proc. of the Amer. Math. Soc., 1999, 127(3): 819-831. MR.1636934 (99g:53064)

[2] Z. H. Hou, Hypersurfaces in a sphere with constant mean curvature, Proc. of the Amer. Math. Soc., 1997, 125(4): 1193-1196. MR.1363169 (97f:53096)

[3] H. W. Xu, On closed minimal submanifolds in pinched Riemannian manifolds, Trans. of Amer. Math. Soc., 1995, 347(5): 1743-1751. MR1243175(95h:53088)

[4] S. S. Chern, M. do Carmo and S. Kobayashi, Minimal submanifolds of a sphere with second fundamental form of constant length, Functional Analysis and Related Fields (F. Broeder, ed.), Springer-Verlag, Berlin, 1970: 59-75. MR0273546 (42:8424)

[5] H. B. Lawson, Jr., Local rigidity theorems for minimal hypersurfaces, Ann. of Math., 1969, 89(2): 187-197. MR0238229 (38:6505)

[6] J. Simons, Minimal varieties in Riemannian manifolds, Ann. of Math., 1968, 88(2): 62-105. MR.0233295 (38:1617)

[7] X. X. Liu and H. Li, Complete hypersurfaces with constant scalar curvature in a sphere, Comm. Math. Univ. Carolinae, 2005, 46(3): 567-575. MR2174533 (2006f:53078)

[8] Q. M. Cheng and H. Nakagawa, Totally umbilic hypersurfaces, Hiroshima Math. J., 1990, 20(4): 1-10. MR.1050421 (91f:53054)

[9] H. W. Xu, Pinching theorems, global pinching theorems and eigenvalues for Riemannian submanifolds, Ph.D. dissertation, Fudan University, 1990.

[10] S. Zhang and B. Wu, Rigidity theorems for complete spacelike hypersurfaces with constant scalar curvature in locally symmetric Lorentz spaces, J. Geom. Phys., 2010, 60: 333-340. MR2587397(2011a:53069)

[11] S. Zhang and B. Wu, Complete hypersurfaces with constant mean curvature in a locally symmetric Riemannian manifold, Acta Math. Scientia, 2010, 30A(4): 1000-1005. MR2722546 (2011j:53112)

[12] S. Y. Cheng and S.T. Yau. Hypersurfaces with constant scalar curvature, Math. Ann., 1977, 225: 195-204. MR0431043 (55:4045)

[13] S. C. Shu and S. Liu, Complete hypersurfaces with constant mean curvature in locally symmetric manifold, Adv. in Math., 2004, 33(5): 563-569. MR2122622 (2006a:53066)

[14] S. Ding and J. Zhang, Hypersurfaces in a locally symmetric manifold with constant mean curvature, Pure Appl. Math., 2006, 22(1): 94-99. MR2258978 (2007e:53072)

[15] H. W. Xu and X. Ren, Close hypersurfaces with constant mean curvature in a symmetric manifold, Osaka J. Math., 2008, 45(2): 747-756. MR2468591 (2009j:53070)

[16] H. Li, Y. Suh and G. Wei, Linear Weingarten hypersurfaces in a unit sphere, Bull. Korean Math. Soc., 2009, 46(2): 321-329. MR2502796 (2010b:53111)

[17] H. Li, Hypersurfaces with constant scalar curvature in space forms, Math. Ann., 1996, 305: 665-672. MR:1399710(97i:53073) 
[18] S. T. Yau, Submanifolds with constant mean curvature, I, II, Amer. J. Math., 1974, 96 : 346-366, 1975, 97: 76-100. MR0370443 (51:6670)

[19] M. Okumura, Hypersurfaces and a pinching problem on the second fundamental tensor, Amer. J. Math., 1974, 96(4): 207-213. MR0353216 (50:5701)

[20] H. Omori, Isometric immersions of Riemannian manifolds, J. Math. Soc. Japan., 19 (1967): 205-214. MR0215259(35:6101)

School of Mathematics, Jiangsu Normal University, Xuzhou, Jiangsu 221116, People's RePublic OF CHINA

E-mail address: zhangshicheng@jsnu.edu.cn

School of Mathematics, Jiangsu Normal University, Xuzhou, Jiangsu 221116, People's Republic of China

E-mail address: wubaoqiang@jsnu.edu.cn 